\title{
The Experiment Research on Giant Magnetoresistance Based on Data Analysis of SPSS
}

\author{
Shuhong Tang * \\ School of Information, Hunan University of Humanities, Science and Technology, Loudi, Hunan \\ 417000, People's Republic of China
}

\begin{abstract}
The basic principles of GMR is introduced and the characteristics of the sensor of GMR is studied by experiment. SPSS is put to use to do data analysis. The results indicate that there is the cubic relationship between the output voltage of the sensor and the external magnetic field and between the output voltage of the sensor and the angle between the sensitive axis of the sensor and the external magnetic field. What's more, the linear relationship between the output voltage and working output voltage of the sensor is proved and the fitting curves of the models and the optimal model equation are acquired.
\end{abstract}

Keywords: Giant Magnetoresistance; the sensor of Giant Magnetoresistance; SPSS

\section{Introduction}

In 1986,P.Grunberg et al observed a phenomenon of antiferromagnetic interlayer coupling when they studied the coupling process of Fe layers across $\mathrm{Cr}$ interlayers [1], which laid a physical foundation for the discovery of Giant Magnetoresistance. In 1988, A.Fert's research group first discovered Giant Magnetoresistance in Fe layers across $\mathrm{Cr}$ interlayers [2]. And since then the in-depth study of Giant Magnetoresistance not only has rapidly promoted the formation and development of a new branch of science--Spintronics, but also contributed to the development and application of material of magnetoresistance and devices of Spintronics. The magnetic read head products and magnetic hard disks based on Giant Magnetoresistance have already been widely applied in many electronic products such as network severs, computers and digital cameras, significantly promoting the computing and information technology; with the advantages of small volume, low power and strong reliability and so on, sensors made from material of giant magnetoresistance are able to sense weak magnetic fields and widely used in bioscience, automobile industry, satellite positioning, household appliances and technology of precision measurement, Giant Magnetoresistance brought revolutionary updates to the development of science and technology and exerted far-reaching influence on human cultural life. Therefore, in the science teaching system of colleges and universities, it is necessary to set up the GMR experiments and further study the GMR and its application by the conclusions by the analysis of experimental data. Putting SPASS into use to process data, which is capable of showing the relevance of each variable, comparing each models and choosing the optimal model equation, this experiment is a way to further research GMR and the character and application of the sensor of the Giant Magnetoresistance.

\section{The basic principle of the experiment}

The theory of Giant Magnetoresistance 
Embodying quantum mechanical effects GMR refers to the phenomenon that the resistivity of magnetic materials changes greatly at applied magnetic field and it is produced at the layered magnetic membrane structure[3,4], This kind of membrane is magnetic multilayer which is a result of the alternate growth of all kinds of magnetic materials and nonmagnetic materials.

With regard to the theory of GMR[5], it can be qualitatively explained by two-fluid model. In this model, conduction electrons are divided into two kinds of categories: carriers spinning up and carriers spinning down. Conduction electrons will receive weaker scattering and lower resistivity if the direction of spin magnetic moment is parallel to the magnetization direction of the area. In the antiferromagnetic coupling of the magnetic moment of the adjacent magnetic layers at magnetic multilayer, conduction electrons spinning up and down receive periodically strong and weak scattering respectively in the transmission procedure. Hence, the magnetic multilayer will be in the high impedance state, as shown in figure 1; When the adjacent magnetic layers at magnetic multilayer tend to parallel under the influence of the applied magnetic field, the conduction electrons spinning up will receive weaker scattering, constituting low-resistance path $\mathrm{R}_{1}$, while the conduction electrons spinning down will constitute high-resistance path $\mathrm{R}_{2}$ because of the stronger scattering. There are half of the electrons in the low-resistance path, leading to the low impedance state of magnetic multilayer, as shown in figure $2[6,7]$.
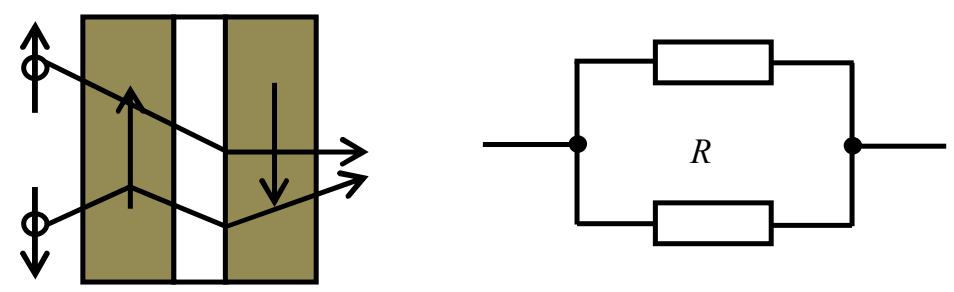

Fig.1. Magnetic moments of magnetic layers arrange antiparallelly, being in high impedance
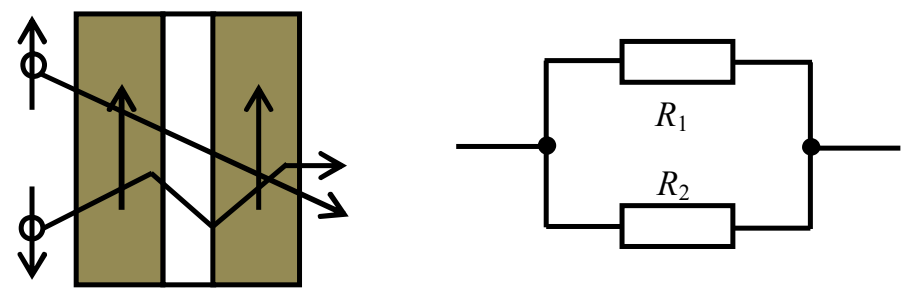

Fig.2. Magnetic moments of magnetic layers arrange parallelly, being in low

The operating principle of the sensor of GMR

The sensor of GMR used in the experiment is Wheatstone bridge consisted of four identical GMRs, as shown in figure 3. Thereinto, R2, R4 are protected by magnetic material. 


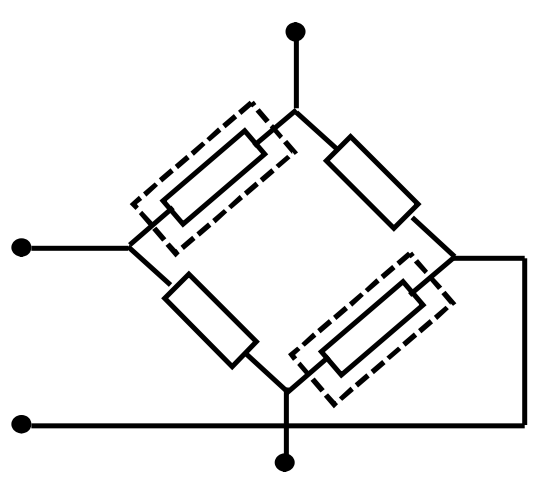

Fig.3. the structure principle of the sensor of GMR

We can work out the output voltage of the sensor easily

$$
U=U_{\text {out }+}-U_{\text {out- }}=\left(V_{+} \cdot \frac{R_{1}}{R_{2}+R_{1}}\right)-\left(V_{+} \cdot \frac{R_{4}}{R_{3}+R_{4}}\right) .
$$

If there is no external magnetic field, $U=0$. Conversely, the resistance of $R_{1} 、 R_{3}-G M R$ without protection will decline while that of $\mathrm{R}_{2} 、 \mathrm{R}_{4}-\mathrm{GMR}$ protected by magnetic material remain unchanged, and then

$$
\begin{aligned}
U=U_{\text {out }+}-U_{\text {out- }} & =V_{+} \cdot \frac{R_{1}-R_{4}}{R_{2}+R_{1}}=-V_{+} \cdot \frac{\Delta R}{2 R+\Delta R} \\
\frac{\Delta R}{R} & =\frac{-2 U}{V_{+}+U}
\end{aligned}
$$

Thereinto, $\Delta R$ is the variation of resistance, and $V_{+}$is the voltage added on the sensor. It can be seen that with the same field strength, the output voltage of the sensor is proportional to the working voltage of the sensor.

Different magnetic fields correspond to different magneto-resistor, producing diverse output voltages, which shows that there is a one-to-one correspondence between the output voltage of the sensor and magnetic fields. The experiment employed current-carrying circular coils (Helmhohz coil) to produce the magnetic field, and according to Biot-savart law, it can be easily figured out the density of magnetic induction of some point which is in the central axis of the current-carrying circular coils

$$
B=\frac{1}{2} \mu_{0} N I R^{2}\left\{\left[R^{2}+\left(\frac{R}{2}+x\right)^{2}\right]^{-\frac{3}{2}}+\left[R^{2}+\left(\frac{R}{2}-x\right)^{2}\right]^{-\frac{3}{2}}\right\} .
$$

The density of magnetic induction of the center of the axis is

$$
B_{0}=\frac{8}{5^{3 / 2}} \times \frac{\mu_{0} N I}{R} \text {. }
$$

Thereinto, $\mu_{0}$ is permeability of vacuum, $N$ is the number of turn coils, $I$ is excitation current, and $R$ is the radius of the coil.

Thus, we can research the relevance between GMR and density of magnetic induction by changing the current of the Helmhohz coil and study the character of the sensor of GMR. 


\section{The introduction of the instruments}

As shown in figure 4, the experimental instruments consist of five parts.

(1)The working voltage of the sensor: Direct current power supply provides the voltage of $1.5 \mathrm{~V}-12 \mathrm{~V}$ continuously and adjustably;

(2)The sensor of GMR: The range of measured value of the magnetic field is $1.5 \mathrm{Gs}-10.5 \mathrm{Gs}$, and the saturation of the field strength is $15 \mathrm{Gs}$;

(3)The standard magnetic field: It includes the constant current source used for the coils whose output current is $0-0.8 \mathrm{~A}$ and continuously adjustable and Helmhohz coil (the number of each turn coil $\mathrm{N}$ is 200 circles, the radius is $10 \mathrm{~cm}$ );

(4)The direct current digital voltmeter used for measuring the output voltage of the sensor;

(5)The direct-current power measured can output current $0-10 \mathrm{~A}$ and is continuously adjustable.

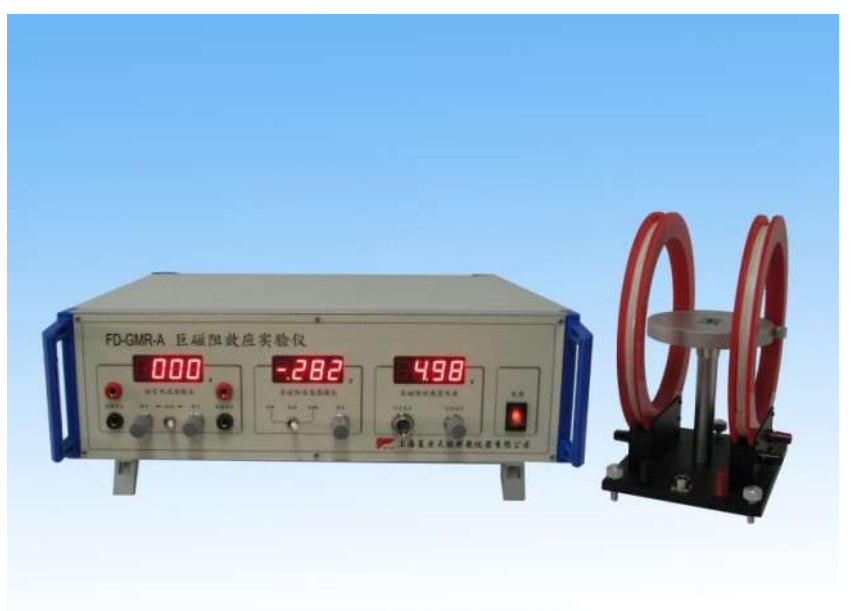

Fig.4. the experimental instruments of GMR

\section{Analysis of experimental results}

This experiment utilizes SPSS to analyze data.

Result analysis of the relation between the output voltage of the sensor and the coil current

Table 1 shows the model summary and parameter estimates of the relevance of the output voltage of the sensor $U$ and the Helmhohz coil current when the working voltage of the sensor is 5V. Figure 5 illustates three fitting curves of the models of the relevance of the output voltage $U$ and the current I. The goodness of fit of linearity, quadratic term and cube can be seen from the table, among which the $\mathrm{R}$ square of cube is highest. Meanwhile, judging from the check result, the significance of the models are all less than 0.01 , illustrating the statistical significance is prominent, so we can get the equation of the optional model--cubic model

$$
U=0.002+0.713 I+0.767 I^{2}-0.616 I^{3} .
$$

(6) Combing with

formula (5), we can know that within limits, there is a cubic relationship between the output voltage of the sensor and external magnetic field. And we also get the relevance between the relatively change rate of GMR $\Delta R / R$ and the induction intensity of external magnetic field $B$ through formula (3), (5) and (6). According to this theory, we are able to get scaling of the sensor of the GMR, measuring the magnetic field produced by the current-carrying conductor. 
Table 1. Model Summary and Parameter Estimates

Dependent Variable: output voltage (V)

\begin{tabular}{|l|r|r|r|r|r|r|r|r|r|}
\hline & \multicolumn{4}{|c|}{ Model Summary } & \multicolumn{4}{c|}{ Parameter Estimates } \\
\cline { 2 - 11 } Equation & R Square & \multicolumn{1}{|c|}{$\mathrm{F}$} & $\mathrm{df1}$ & \multicolumn{1}{c|}{$\mathrm{df} 2$} & \multicolumn{1}{c|}{ Sig. } & Constant & \multicolumn{1}{c|}{ b1 } & \multicolumn{1}{c|}{$\mathrm{b} 2$} & $\mathrm{~b} 3$ \\
\hline Linear & .999 & 16061.086 & 1 & 15 & .000 & -.014 & .965 & & \\
Quadratic & .999 & 7802.563 & 2 & 14 & .000 & -.011 & .943 & .027 & \\
Cubic & 1.000 & 180386.846 & 3 & 13 & .000 & .002 & .713 & .767 & -.616 \\
\hline
\end{tabular}

The independent variable is coil current $(\mathrm{A})$.

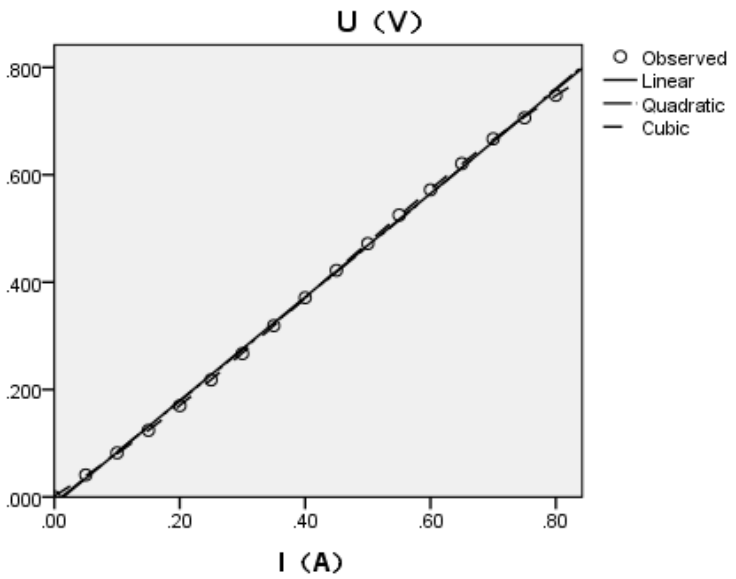

Fig.5. fitting curves of output voltage and coil current

Result analysis of the relationship between output voltage of the sensor and the angle between the sensitive axis of the sensor and the external magnetic field

Table 2. Model Summary and Parameter Estimates

Dependent Variable: output voltage (V)

\begin{tabular}{|l|r|r|r|r|r|r|r|r|r|}
\hline & \multicolumn{4}{|c|}{ Model Summary } & \multicolumn{4}{c|}{ Parameter Estimates } \\
\cline { 2 - 10 } Equation & R Square & \multicolumn{1}{|c|}{$\mathrm{F}$} & $\mathrm{df1}$ & \multicolumn{1}{c|}{$\mathrm{df} 2$} & \multicolumn{1}{c|}{ Sig. } & Constant & $\mathrm{b} 1$ & $\mathrm{~b} 2$ & $\mathrm{~b} 3$ \\
\hline Linear & .965 & 474.238 & 1 & 17 & .000 & 1.318 & -.780 & & \\
Quadratic & .997 & 2574.432 & 2 & 16 & .000 & 1.189 & -.260 & -.331 & \\
Cubic & 1.000 & 69617.074 & 3 & 15 & .000 & 1.149 & .096 & -.913 & .247 \\
\hline
\end{tabular}

The independent variable is the Angle ( $\mathrm{rad}$ ).

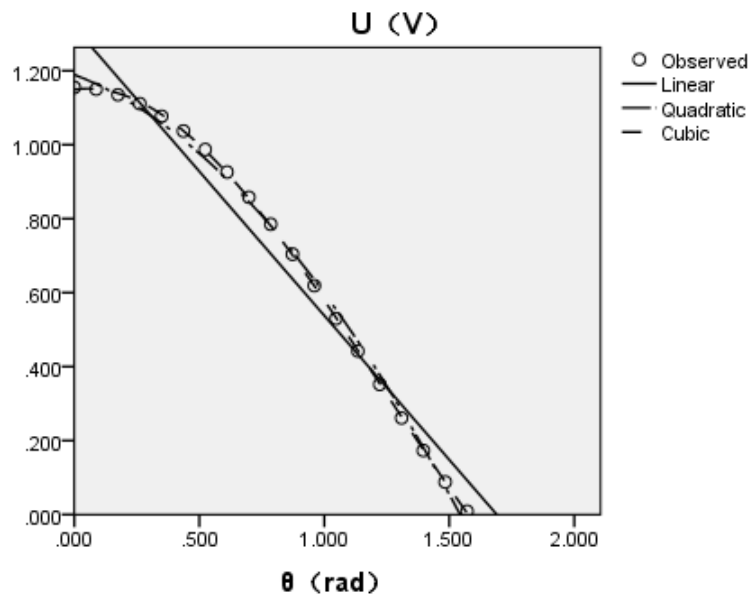

Fig.6. fitting curves of output voltage and the angle 
Table 2 shows the model summary and parameter estimates of the relevance of the output voltage of the sensor $U$ and the angle between the sensitive axis of the sensor and the external magnetic field $\theta$ when the working voltage of the sensor is $12 \mathrm{~V}$ and the coil current is $0.6 \mathrm{~A}$. Figure 6 shows three fitting curves of the models of the relevance of the output voltage and the angle. We can know from the table that the $\mathrm{R}$ square of cube is highest. Meanwhile, judging from the check result, the significance of the models are all less than 0.01 , illustrating the statistical significance is prominent, so we can get the equation of the optional model

$$
U=1.149+0.096 \theta-0.913 \theta^{2}+0.247 \theta^{3} .
$$

It can be seen that when the working voltage and the coil current remain unchanged and the direction of the external magnetic field is parallel to that of the sensitive axis of the sensor, the output voltage of the sensor will reach maximum. And as the angle increases from $0^{\circ}$ to $90^{\circ}$, the output voltage decreases gradually. What's more, the relationship between the output voltage and the angle is cubic relationship. Accordingly, the sensor of GMR can be applied to navigation. That is to say, when the direction of transportation changes during driving, the angle between the sensor of GMR and geomagnetic field will changes as well, which will cause changes of resistance of the sensor and the output voltage. So, we can confirm the locations of cars, steamships and so on by the changing situations. Besides, the sensor of GMR can also be employed to detect the substance with magnetic characteristics.

Result analysis of the relationship between output voltage and working voltage of the sensor

Table 3 shows the model summary and parameter estimates of the linear relevance of the output voltage and the working voltage of the sensor when the coil current is $0.6 \mathrm{~A}$. Figure 7 is the fitting curves of the output voltage and the working voltage of the sensor. Judging from the check result, the significance of the model is less than 0.01 and passes examination. We can get the equation of the linear model

$$
U=-0.005+0.116 E
$$

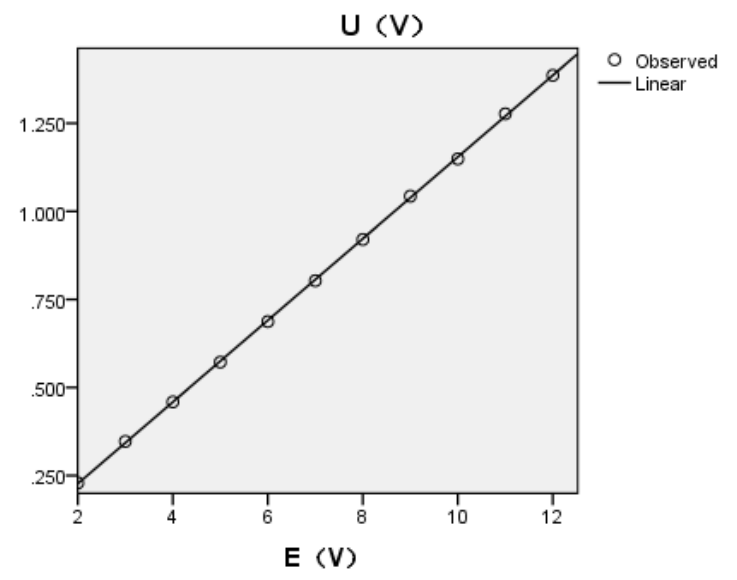

Fig.7. fitting curve of output voltage $U$ and working voltage $E$

\begin{tabular}{|c|c|c|c|c|c|c|c|}
\hline \multicolumn{3}{|c|}{ Dependent Variable: } & \multicolumn{5}{|c|}{$(\mathrm{V})$} \\
\hline \multirow[b]{2}{*}{ Equation } & \multicolumn{5}{|c|}{ Model Summary } & \multicolumn{2}{|c|}{ Parameter Estimates } \\
\hline & R Square & $\mathrm{F}$ & $\mathrm{df1}$ & $\mathrm{df} 2$ & Sig. & Constant & b1 \\
\hline Linear & 1.000 & 85359.539 & 1 & 9 & .000 & -.005 & .116 \\
\hline
\end{tabular}

Table 3. Model Summary and Parameter Estimates 
It can be seen that when the external magnetic field stay unchanged, the greater the working voltage of the sensor of GMR is, the grater the output voltage is, which means the more sensitive the sensor is.

\section{Conclusion}

According to the theory of GMR, we can utilize Wheatstone bridge to make the sensor of GMR to do the relevant experiments which are analyzed by SPSS and therefore get to the conclusions: There is a cubic relationship between the output voltage of the sensor and external magnetic field; When the working voltage and the coil current remain unchanged and the angle between the external magnetic field and the sensitive axis of the sensor increases from $0^{\circ}$ to $90^{\circ}$, the output voltage decreases gradually and there is a cubic relationship between the output voltage and the angle; When the external magnetic field stay unchanged, there is a linear relationship between the output voltage and the working voltage of the sensor, and the greater the working voltage of the sensor of GMR is, the more sensitive the sensor is.

Meanwhile, it can be seen that the function of data processing is powerful if using SPSS to analyze the experiment of GMR. SPSS is able to analyze the relevance of all variables, compare all kinds of fitted models and choose the optimal model scientifically, which can guarantee the high precision of the results of data processing.

\section{Reference}

[1] P. Grunberg, R. Schreiber, Y. Pang. Layered magnetic structures: Evidence for antiferromagnetic coupling of Fe layers across $\mathrm{Cr}$ interlayers[J]. Phys. Rev. Lett, 1986, 57(19):2442.

[2] M. N. Baibich, J. N. Broto, A. Fert, et al. Giant magnetoresistance of $(001) \mathrm{Fe} /(001) \mathrm{Cr}$ magnetic superlattices[J]. Phys. Rev. Lett, 1988, 61(21):2472.

[3] P. B. Allen, Magnetism and magnetoresistance in magnetic multilayers[J].Solid State Communications, 1997, 102(2-3): 127 134.

[4] T. R. Mcguire, R. I. POTTER. Anisotropic magnetoresistance in ferromagnetic 3d alloys[J]. IEEE Transactions on Magnetics,1975,11(4):1018.

[5] S. M. Thompson. The discovery,development and future of GMR:The Nobel Prize 2007[J]. J.phys.D,2008,41:093001.

[6] E. Y. Tsymbal, D. G. Pettifor. Perspectives of Giant Magnetoresistance[J]. Solid State Physics, 2001, 56: 113 119.

[7] N. Strelkov, A. Vedyaev, B. Dieny. Extension of the semiclassical theory of current-perpendicular-to-plane giant magnetoresistance including spin flip to any multilayered magnetic structures[J]. Journal of Applied Physics, 2003, 94(5) : 3278 3287. 